\title{
Acute mesenteric ischaemia : Part II
}

\author{
Anton S. Dias Perera ${ }^{1,2}$, Michael J. Rohrer ${ }^{2}$ \\ 1Baptist Memorial Hospital, Memphis, Tennessee, USA. \\ ${ }^{2}$ Division of Vascular Surgery, University of Tennessee, USA.
}

\begin{abstract}
Editor's note:
This is the second part of a review on acute mesenteric ischaemia. The first part is published in the current issue on pages 18-23 (http://doi.org/10.4038/sljs.v36i1.8476). Readers are strongly recommended to read these two articles in continuity."
\end{abstract}

\section{Treatment}

Some general principles of treatment apply to all cases of acute mesenteric ischaemia: the importance of early diagnosis, prompt exploratory laparotomy, adequate restoration of arterial perfusion, resection of non-viable bowel, second look laparotomy and supportive intensive postoperative care.

A soon as diagnosis of AMI is established or suspected, fluid resuscitation should be begun immediately with isotonic crystalloids, blood and blood products as deemed necessary. Electrolyte imbalances such as hyperkalemia, acidosis and other metabolic abnormalities should be corrected. Invasive arterial and central venous pressure monitoring along with monitoring of urine output are recommended to ensure adequate optimization prior to intervention. Broad-spectrum antibiotics are given to minimize the consequences of bacterial translocation and systemic sepsis. Intravenous heparin should be administered unless there is a specific contraindication. Fluid resuscitation should be adjusted until adequate right-sided filling pressures are achieved. Vasopressors may be needed to maintain systemic blood pressure at physiological levels $(>100 \mathrm{~mm} \mathrm{Hg})$; however, this should not substituted for inadequate volume resuscitation.

\section{Surgical exploration}

Exploratory laparotomy is required in all patients with clinical suspicion of threatened bowel. A midline, vertical incision is preferred given that it is expeditiously performed and allows the flexibility to thoroughly assess the entire GI tract and the vascular territories of interest. Furthermore, this allows great flexibility for the placement intestinal stomas as

Correspondence: Anton S. Dias Perera

E-mail: surathdp@yahoo.com

Received: 13-12-2017 Accepted: 18-04-2018

(iD) http://orcid.org/0000-0001-6288-5121

DOI:http://doi.org/10.4038/sljs.v36i1.8477 needed. Lower extremities should be prepped for possible saphenous vein harvest.

Intra-operative evaluation of bowel viability can be difficult in the setting of AMI. Differentiation of irreversible necrosis from potentially salvageable but severely ischemic bowel is often difficult prior to revascularization and therefore best left for re-evaluation following restoration of blood flow. Areas of obvious necrosis or perforation causing spillage should be resected expeditiously without re-anastomosis. Final assessment of bowel viability must be reserved until after completion of revascularization.

There are several ancillary techniques available for assessing viability of bowel. Hand-held Doppler ultrasound probe (continuous wave 9-10 MHz) can be used to detect pulsatile flow signals. Although it is a useful adjunct to clinical evaluation alone, it may lack sensitivity. Other useful adjuncts include photophlethysmography, injection of fluorescein dye and the use of a perfusion fluorometer to detect flow. Whitehill et al, in a study published in 1988, found that thresh-old blood flow detection by any one of these methods, especially fluorescein, occurs at levels inadequate to guarantee tissue viability [1]. This means ischemic tissue damage may occur in spite of detectable blood flow using these adjuncts. The ultimate decision regarding bowel viability therefore lies in the sound clinical judgment of an experienced operating surgeon. Treatment algorithms that include mandatory second-look laparotomy allow re-evaluation of bowel following revascularization and adequate resuscitation, thereby providing the opportunity to re-anastomose the bowel ends that were divided during intestinal resection of the index operation.

An important principle of bowel resection in AMI is to minimize disruption of mesenteric vascular arcades by carrying out segmental resection while sparing as much of the mesentery as possible. This can be accomplished easily by using devices such as LigasureTM (Medtronic Inc., Minneapolis, MS, USA) or with standard ligate and divide technique. This approach should be contrasted with conventional wedge shaped resection undertaken during routine small bowel operations. 


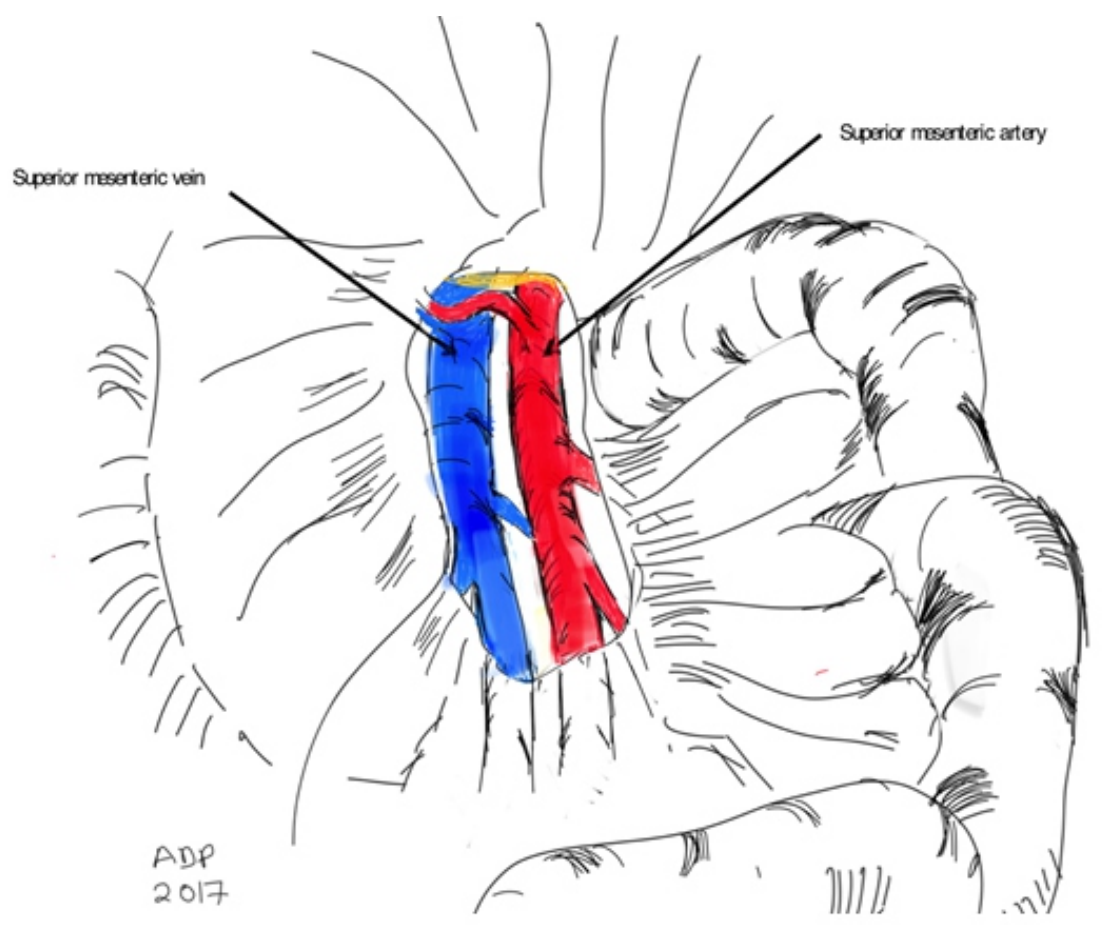

figure 5. Anterior approach to the SMA for SMA embolectomy or bypass from a supraceliac inflow source

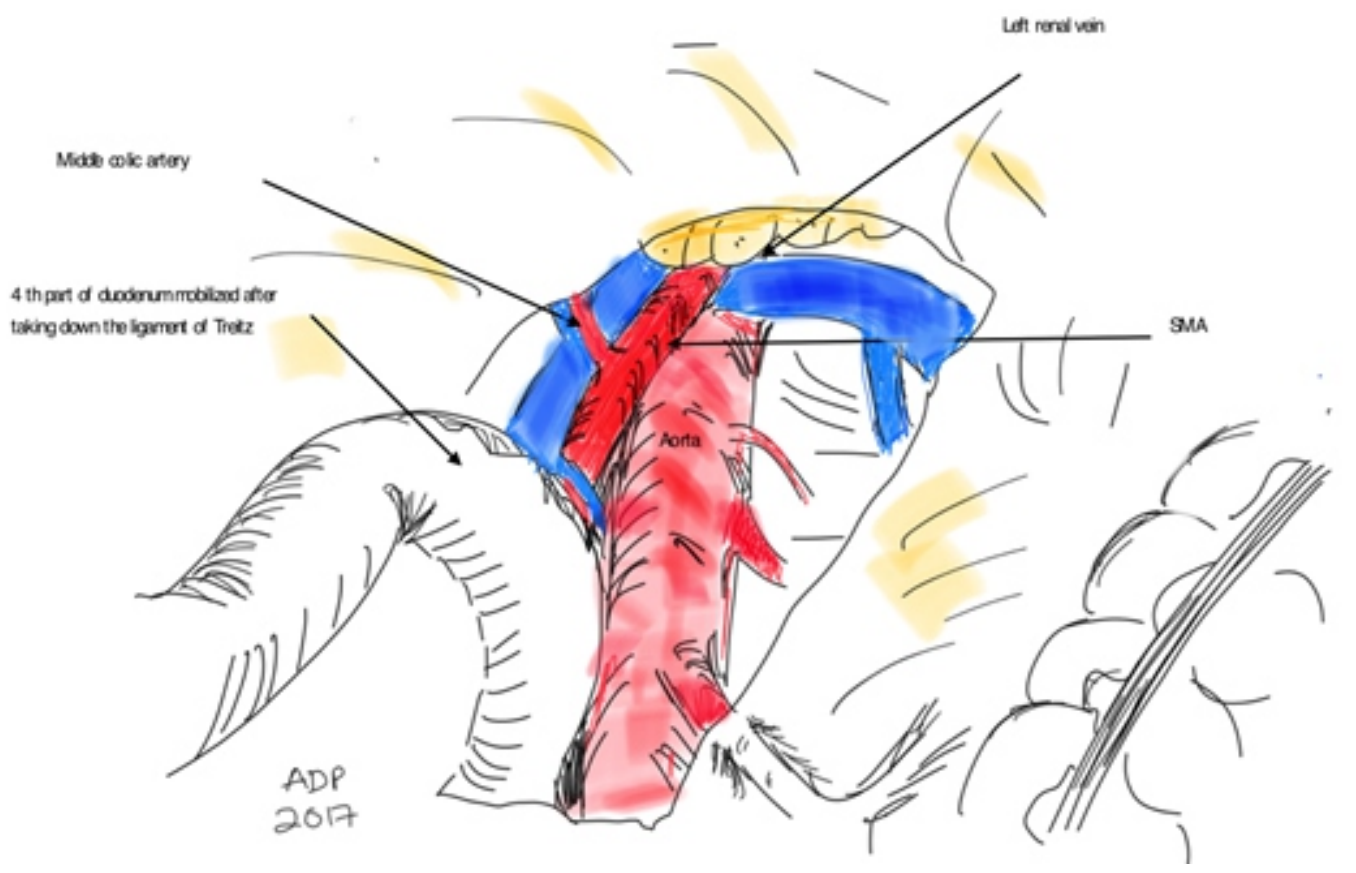

Figure 6. Lateral approach to SMA for bypass

\section{Open surgical revascularization}

There are two standard approaches to the surgical exposure of the SMA: anterior and lateral. The anterior approach near the root of the mesentery is most suitable for embolectomy whereas the lateral approach, with mobilization of the fourth part of duodenum and ligament of Treitz, is often preferred for arterial bypass (figures 5,6). Selection between these two approaches depends on the diagnosis (embolus versus thrombosis) and the need for complex arterial reconstruction.

\section{Embolectomy}

Superior mesenteric artery embolectomy can be accomplished easily through an anterior exposure with elevation of transverse mesocolon superiorly and retraction of small bowel to the patient's right. The pulseless mesenteric vascular bundle can be palpated and exposed by incising the peritoneum at the base of the transverse mesocolon. Multiple venous tributaries, lymphatics and autonomic nerve fibers encase the mesenteric vascular bundle. The SMA lies to the left of the superior mesenteric vein, and careful dissection is 
needed for segmental exposure of the SMA from middle colic to right colic branches. Following systemic heparinization, the arteriotomy can be either transverse or longitudinal depending on the vessel diameter but in the case of longitudinal arteriotomy, patch closure (saphenous vein) is required. A transverse arteriotomy is technically easier to perform and may be used if the vessel diameter is at least 6 $\mathrm{mm}$. An interrupted suture technique using non-absorbable, monofilament suture (6-0 Prolene) is recommended for closure to prevent stenosis.

Proximal embolectomy is performed using 3Fr or 4Fr Fogarty catheters. Brisk, pulsatile flow is expected following extraction of embolus. For distal thrombo-embolectomy, $2 \mathrm{Fr}$ or $3 \mathrm{Fr}$ catheters may be used. Distal passage of catheters must be done with care to minimize damage or rupture of distal mesenteric arteries. It is preferred to use air for Fogarty embolectomy catheter balloon inflation, as opposed to saline, since it allows better tactile sensation regarding pressure applied to the vessel wall. Although the major bulk of embolus can be extracted using this technique, thrombus lodged in smaller branches distal to the embolus cannot

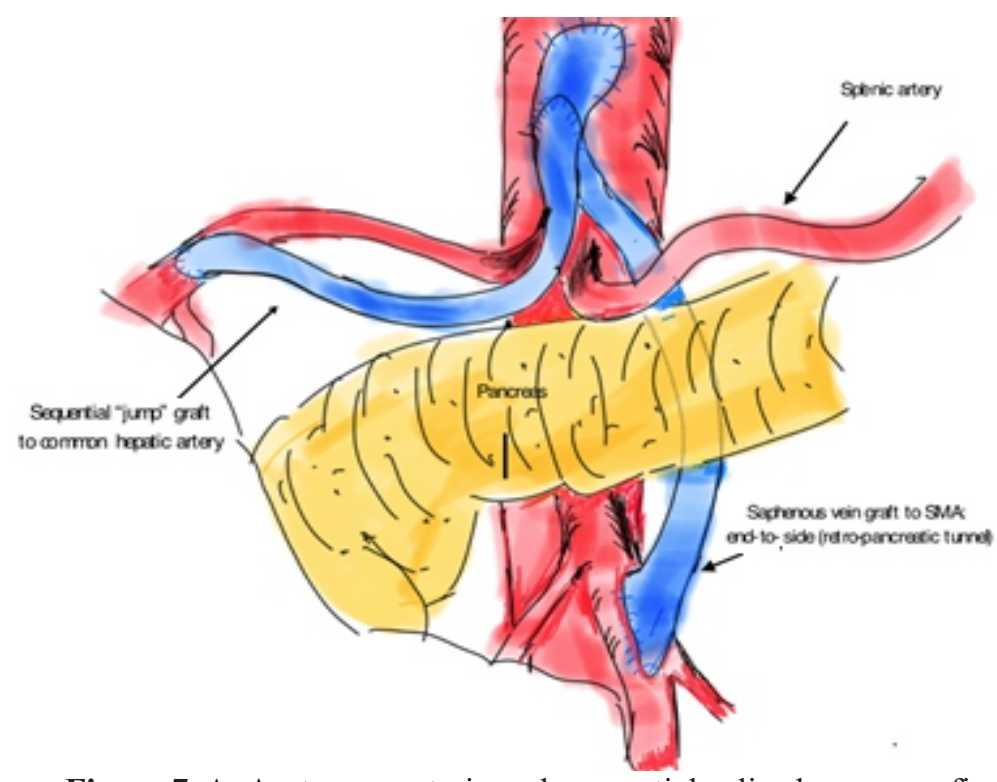

bypass may be performed in the setting of occlusion of both SMA and celiac trunk (Figures 7A,7B). However, revascularization of both celiac and SMA territories is not mandatory in the emergency setting, and a single bypass to the SMA is all that is required [2].

The supra-celiac aorta is usually spared from calcification and intraluminal plaque formation and can be exposed through the lesser omentum after repositioning retractors. The left triangular ligament of the liver is taken down to the left hepatic vein. The presence of a nasogastric tube allows the esophagus to be palpated and retract out of harm's way. The diaphragmatic crura are divided to expose the supra-celiac aorta. A partially occluding clamp, e.g. Satinsky, is often sufficient for proximal anastomosis thus minimizing the physiological stress of supraceliac aortic cross clamping. An antegrade aorto-mesenteric bypass has the advantage of being less prone to kinking and may be tunneled in either retropancreatic or supra-pancreatic position. The use of a prosthetic graft is often convenient, but should be avoided in the setting of intestinal infarction to avoid graft contamination.

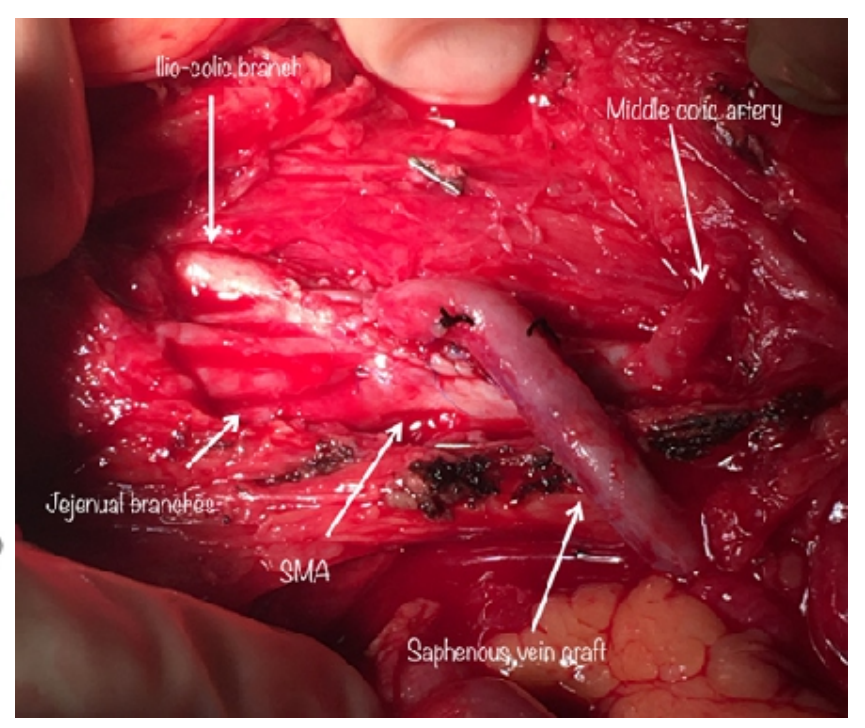

Figure 7 A. Aorto-mesenteric and sequential celiac bypass configuration (retro-pancreatic tunnel for SMA bypass)

B. Distal anastomosis of aorto-superior mesenteric bypass with saphenous vein

always be reliably extracted. Therefore, a recombinant tissue plasminogen activator, $2-5 \mathrm{mg}$ (or an equivalent thrombolytic agent), can be administered distally following final passage of embolectomy catheter, and allowed to dwell for few minutes prior to restoring distal flow.

\section{Surgical bypass}

Options for open reconstruction include a supra celiac aortomesenteric, infra-renal aorto-mesenteric and iliac-tomesenteric artery bypass. If the patient's physiological status permits and adequate conduit is available, aorto-superior mesenteric artery bypass with a sequential celiac branch
Surgical bypass with infra-renal aorta or iliac artery as inflow is best performed via lateral approach to the SMA as discussed previously. Following mobilization of fourth part of the duodenum, peritoneum is exposed lateral to the duodenum and the retroperitoneal aorta is exposed through the same incision. More proximal dissection with gentle retraction of the pancreas facilitates SMA exposure. Several configurations and approaches are available for mesenteric revascularization. Retrograde graft orientation is commonly performed with inflow from the right common iliac artery, forming a "lazy C" configuration (Figure 8). An alternative configuration is to use the saphenous vein with left common 


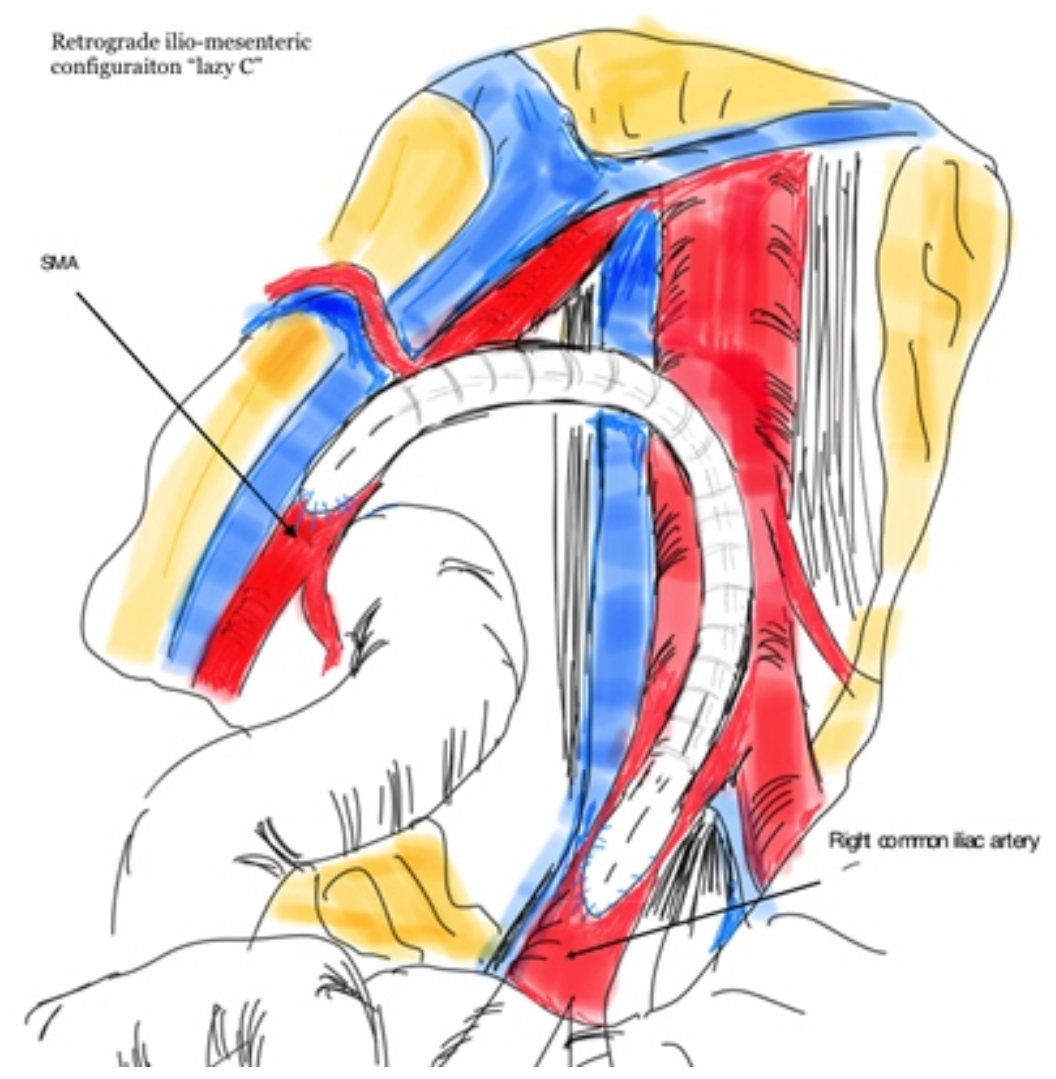

Figure 8. "Lazy C" configuration: ilio-mesenteric bypass with PTFE graft (either right or left iliac artery may be used as inflow)

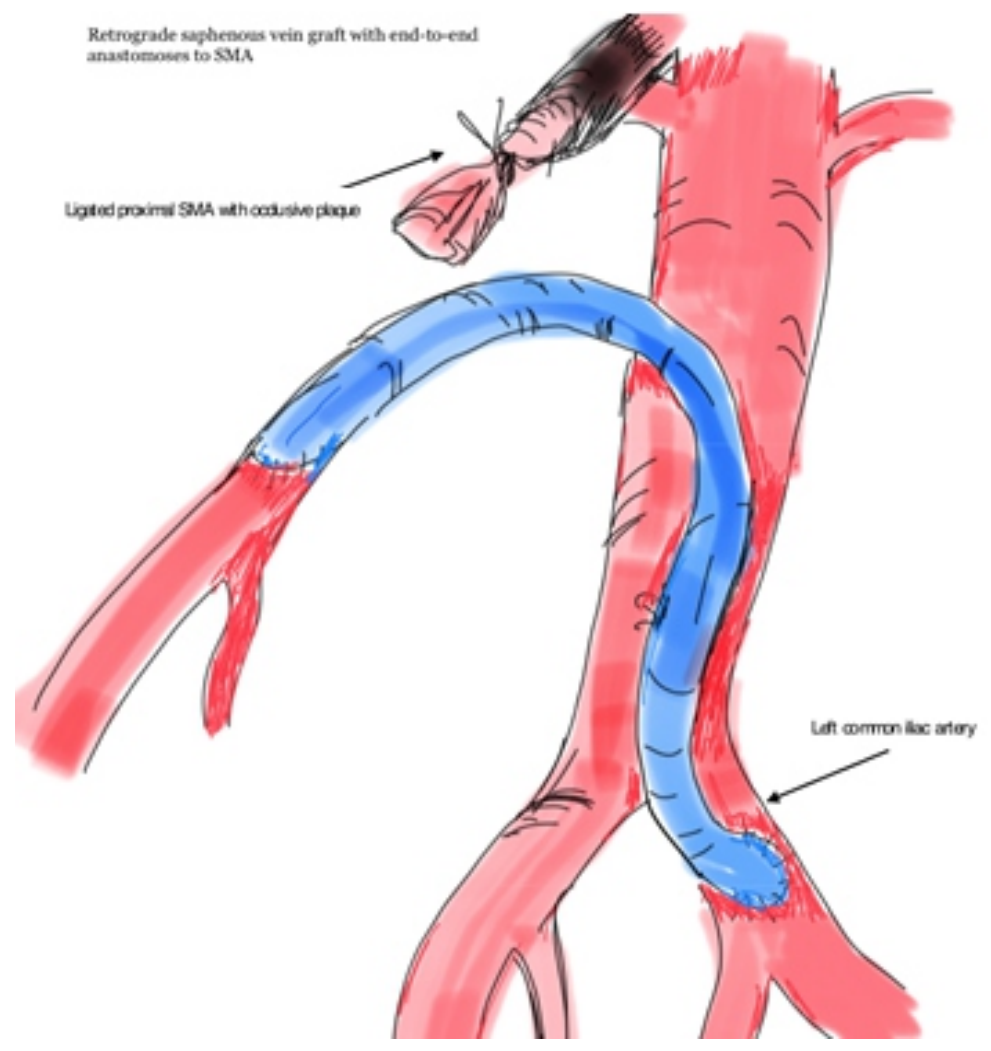

Figure 9. Alternative retrograde bypass with saphenous vein 


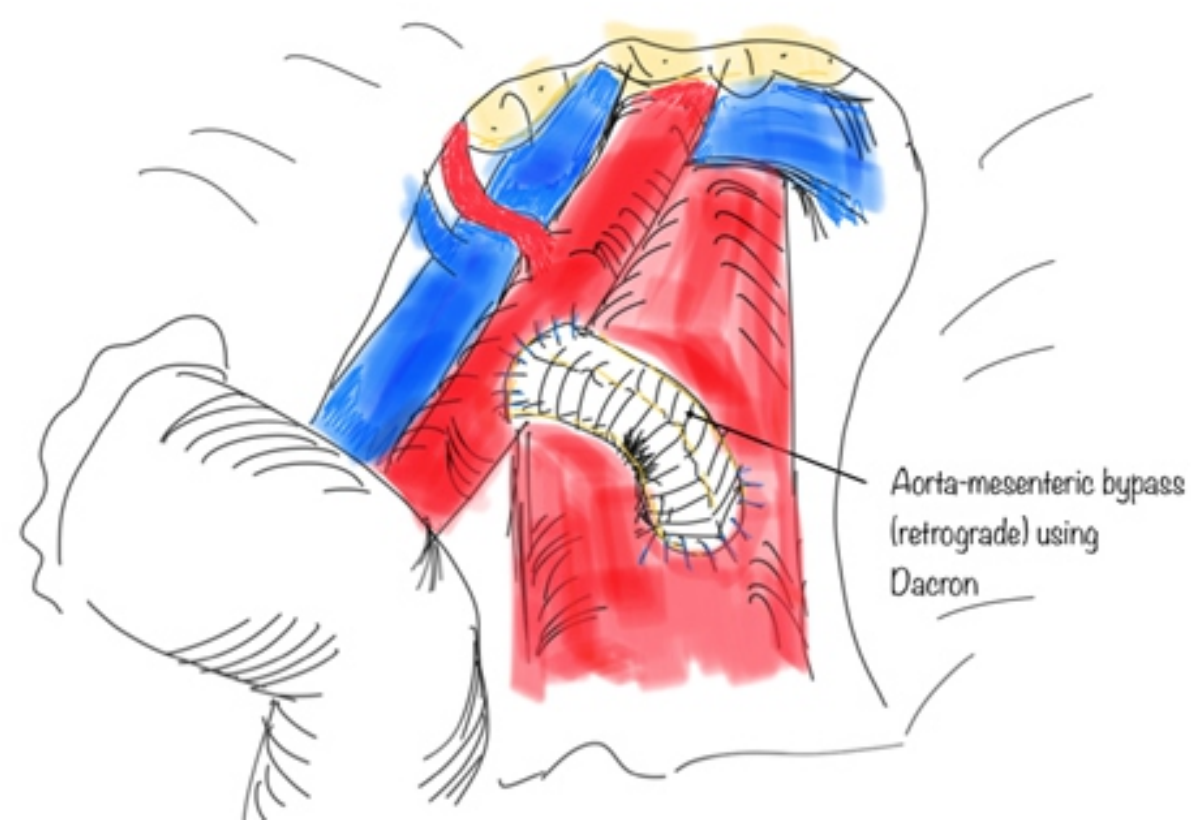

Figure 10. Direct bypass to SMA - may be more prone to kinking

iliac artery or distal infra-renal aorta as inflow vessel (Figure 9). A short aorto-mesenteric bypass configuration can also be used with end-to-side anastomosis to the SMA (Figure 10). In general, the challenge with all retrograde bypass configurations, is to ensure that the graft does not kink when retractors are removed and eviscerated bowel returned to the abdomen. Saphenous vein is the preferred conduit if a good quality vein is available. Alternatively, synthetic grafts such as Dacron or externally supported expanded polytetraflouroethylene (ePTFE) are readily available and provide excellent size choices $(6-8 \mathrm{~mm}$ diameter grafts). Prosthetic graft material should be avoided in the setting of intestinal infarction or bowel spillage.

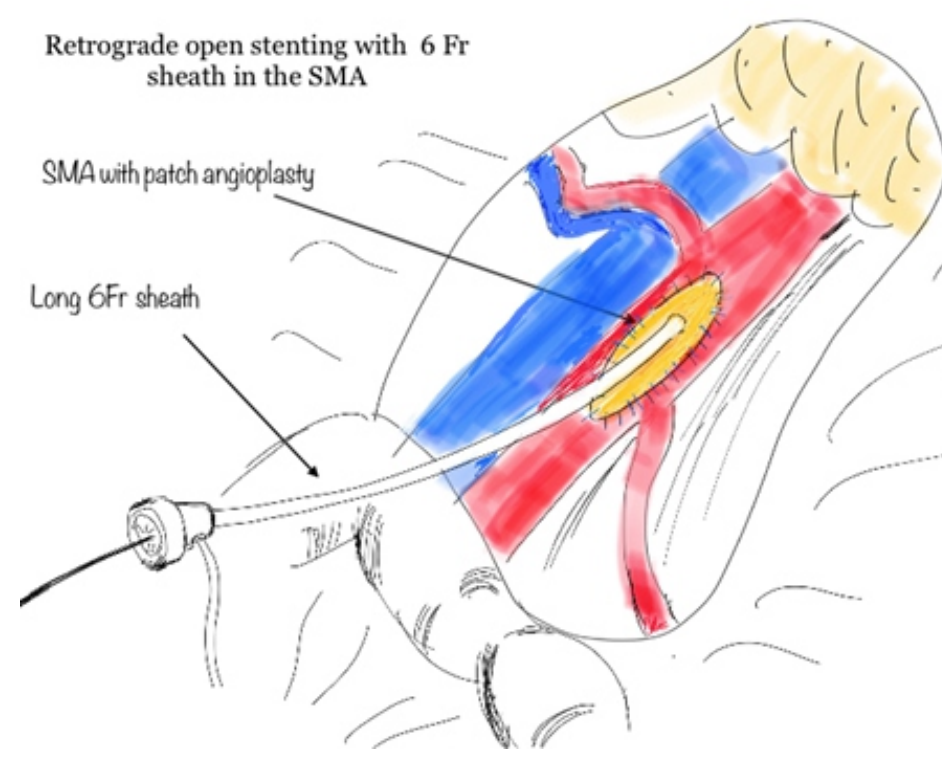

\section{Completion arteriography}

A completion arteriogram may be performed at the surgeon's discretion. Often, this is technically difficult during an open laparotomy and the quality of imaging may be compromised. A completion duplex scan is a valid alternative and is the authors' preferred modality for evaluation of anastomoses.

\section{Hybrid technique}

Hybrid open-endovascular approach, described by Wyers et al from Dartmouth[3], combines endovascular techn-ique during open laparotomy. The SMA is approached via anterior approach and a local thromboendarterectomy and patch angioplasty is performed. The SMA is cannulated retrograde and a long, flexible 6 Fr sheath is directed proximally. Sheath access close to the aorta facilitates crossing the lesion and has a high technical success rate. The SMA is treated with a balloon-expandable stent (Figure 11). This technique has some utility if a donor site is not available for open bypass due to intrinsic disease of aorta or iliac arteries, or when the patient is poor candidate for complex open repair. The patient must be placed on a suitable OR table through which AP and lateral imaging is possible.

\section{Endovascular treatment}

Percutaneous treatment options for the management of acute mesenteric ischaemia are evolving. However, broad applicability of the technique is limited by difficulties in extracting large embolic fragments in the case of SMA embolization and managing chronic occlusions of the SMA in the setting of AMI due to atherosclerotic occlusive disease. Furthermore, a non-operative approach precludes the ability to directly assess bowel perfusion and viability.

Figure 11. Hybrid open-endovascular intervention of SMA 


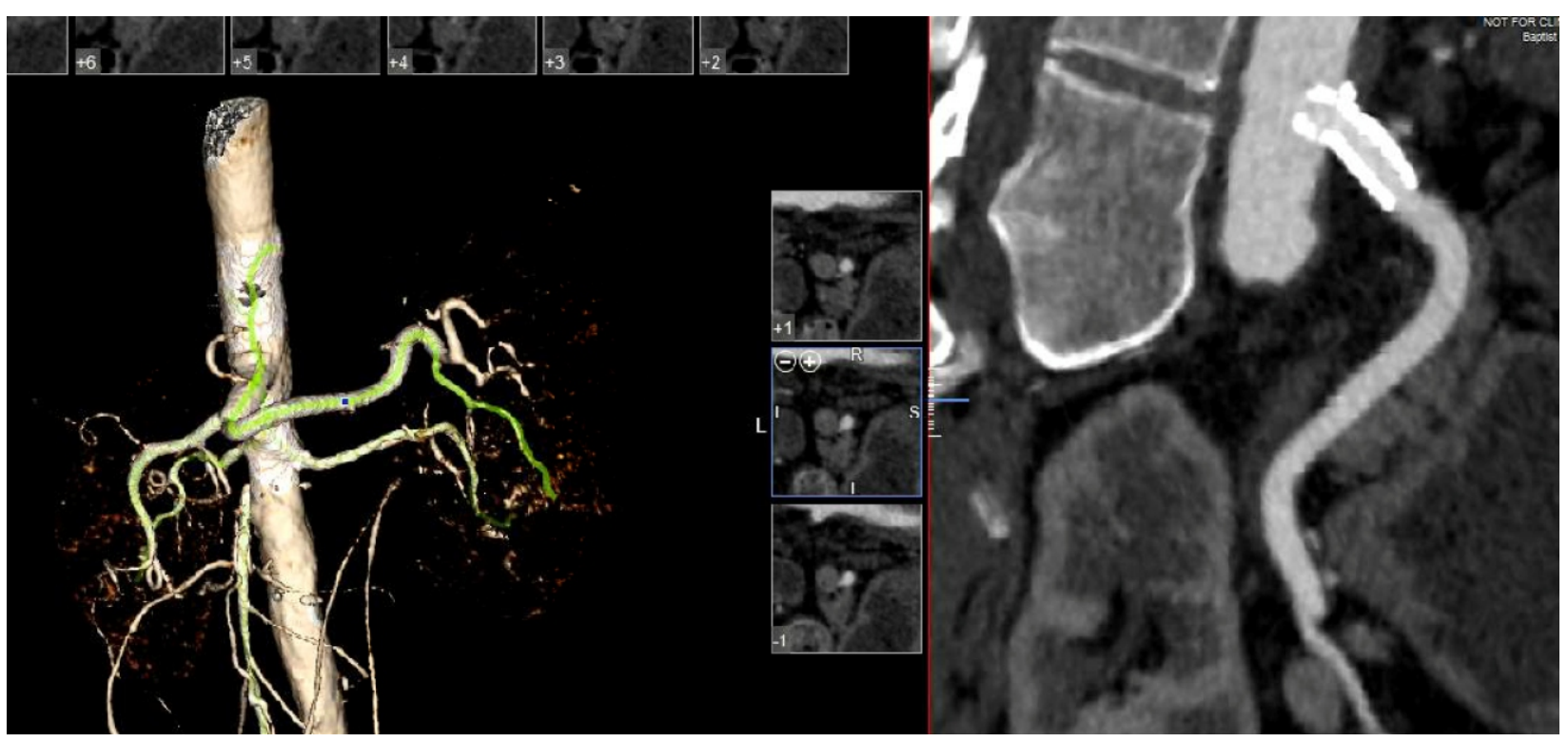

Figure12. Celiac stent in a patient with acute mesenteric ischaemia. Collaterals via the pancreatico-duodenal arcade reconstitute the SMA (arrow)

Endovascular treatment of mesenteric artery embolism may also be constrained by treatment duration required for thrombolysis. Either mechanical or chemical thrombectomy, or a combination of both, have been described using endovascular methods $[4,5,6]$. Majority of patients treated with endovas-cular method still required exploratory laparotomy, under-scoring the need for direct assessment of bowel viability in these patients. In spite of these shortcomings, there are multiple studies showing acceptable outcomes with endovascular intervention [4,5,6] (Figure 12).

\section{Visceral malperfusion from aortic dissection and sponta- neous SMAdissection}

Visceral malperfusion is a catastrophic sequelae of both acute Stanford type A and B dissections. A recent study of the IRAD (International Registry of Acute Dissection) database showed that mortality exceeded $95 \%$ when treatment of type A dissection is undertaken without initial treatment of visceral malperfusion [7]. Therefore, some authors have recommended performing simultaneous mesenteric revascularization procedure and central aortic repair to prevent intestinal infarction resulting from the long duration of ischaemia incurred while repairing the type A dissection [8]. Type B dissections with visceral malperfusion are treated with repair of entry tear, either surgically or with an endograft. Additional intervention is performed as needed to treat persistent malperfusion. Operative techniques include open aortic fenestration and bypass. Endovascular fenestration and stenting are also increasingly used to treat visceral malperfusion syndrome in the setting of dissection. Intravascular ultrasound (IVUS) is an invaluable tool in treatment of aortic dissection. It provides important imaging cues that enable accurate identification of true and false lumen during endovascular intervention.
Acute mesenteric ischaemia presenting as a complication of spontaneous SMA dissection may be treated initially with anticoagulation and using either open or endovascular techniques already described if the clinical condition requires.

Treatment of non-occlusive mesenteric ischaemia (NOMI) Prompt mesenteric arteriography is recommended for patients suspected with NOMI. The angiographic appearance is fairly typical with several specific findings: there is narrowing of the origins of multiple branches of the SMA, alternate dilatation and narrowing of intestinal branches - "string of sausages" sign, and severe spasm of mesenteric arcades, "pruning", resulting in impaired filling of intramural vessels. Injection of intra-arterial vasodilator (Papaverine, $60 \mathrm{mg}$ or nitroglycerin, $400-800 \mathrm{mcg}$ ) can be both diagnostic and therapeutic. A vasodilator infusion via an angiographic catheter placed in the proximal SMA can be started. Infusion rate is titrated according systemic blood pressure. An important technical detail is to ensure that the tip of the catheter and the infusion ports are positioned entirely in the SMA to prevent systemic hypotension from vasodilators 'escaping' to the aorta.

\section{Second-look laparotomy}

Planning for a second look laparotomy is an essential step in the treatment algorithm of acute mesenteric ischaemia. This approach allows the operating surgeon to minimize bowel resection at initial exploration and avoid bowel anastomosis. During the first operation, the resected bowel ends are expeditiously stapled or sewn shut without anastomosis. The abdominal wall may be closed temporarily with the expectation of returning to the operating room in $24-48$ hours. 
At the time of second-look laparotomy, viability of the revascularized bowel is re-assessed and resection of nonviable segments performed as needed. Anastomosis can be completed during this time. Bowel perfusion and patency of mesenteric arteries and bypasses(s) may also be reassessed using manual inspection, hand-held Doppler probe or portable duplex ultra-sound. Occasionally, a third look laparotomy may be required. The guiding principle of treating acute mesenteric ischaemia is to ensure bowel viability before definitive resection and anastomosis.

\section{Post-operative intensive care}

Post-operatively, AMI patients require additional volume resuscitation with crystalloids due to third spacing. Twentyfour hour fluid requirement may be as high 10-15 liters. Serial clinical examinations and trends in leucocyte count and lactate levels are useful to monitor patient's clinical progress. A trend in the wrong direction should prompt further evaluation with an appropriate imaging study (CTA, DSA) and early operative re-exploration.

\section{Outcome}

In spite of numerous advances in surgical and general intensive care, acute mesenteric ischaemia continues to have a high mortality rate. A contemporary, single center experience of acute arterial mesenteric ischaemia from Mayo Clinic reported 30-day mortality rates of $19 \%$ and $17 \%$ for patients presenting with arterial embolism and arterial thrombosis respectively [9]. Early diagnosis, and effective and timely intervention involving excellent surgical and critical care is necessary to achieve survival in an otherwise uniformly fatal diagnosis.

All authors disclose no conflict of interest. The study was conducted in accordance with the ethical standards of the relevant institutional or national ethics committee and the Helsinki Declaration of 1975, as revised in 2000 .

\section{References}

1. Whitehill TA, Pearce WH, Rosales C, Yano T, Van Way CW, Rutherford RB. Detection thresholds of nonocclusive intestinal hypoperfusion by Doppler ultrasound, photoplethysmography, and fluorescein. J Vasc Surg 1988; 8: 28-32. https://doi.org/10.1067/mva.1988.avs0080028.

2. Foley Matthew I, Moneta Gregory L, Abou-Zamzam Jr, Ahmed M, Edwards JM, Taylor jr, LM, Yeager RA, Porter JM. Revascularization of the superior mesenteric artery alone for treatment of intestinal ischemia. J Vasc Surg 2000;32:37-47. https://doi.org/10.1067/mva.2000.107314.

3. Wyers MC, Powell RJ, Nolan BW, Cronenwett JL. Retrograde mesenteric stenting during laparotomy for acute mesenteric ischemia. J Vasc Surg; 2007; 45:269-75. https://doi.org/10.1016/j.jvs.2006.10.047.

4. Simó G, Echenagusia AJ, Camúñez F, Turégano F, Cabrera A, Urbano J. Superior mesenteric arterial embolism: local fibrinolytic treatment with urokinase. Radiology [Internet]. Radiological Society of North America (RSNA); 1997 Sep; 204(3):775-9. https://doi.org/10.1148/radiology.204.3.9280258.

5. Raupach J, Lojik M, Chovanec V, Renc O, Strýček M, Dvořák P, Hoffmann P et al. Endovascular management of acute embolic occlusion of the superior mesenteric artery: a 12-year singlecentre experience. Cardiovascular and Interventional Radiology, 2016;2:195-203.

https://doi.org/10.1007/s00270-015-1156-6

6. Arthurs ZM, Titus J, Bannazadeh M, Eagleton MJ, Srivastava S, Sarac TP, Clair DG. A comparison of endovascular revascularization with traditional therapy for the treatment of acute mesenteric ischemia. J Vasc Surg. 2011;53(3):698-704. https://doi.org/10.1016/j.jvs.2010.09.049.

7. Eusanio MD, Trimarchi S, Patel HJ, Hutchison S, Suzuki T, Peterson MD, Di Bartolomeo R, Folesani G, Pyeritz RE, Braverman AC, Montgomery DG, Isselbacher EM, Nienaber CA, Eagle KA, Fattori R. Clinical presentation, management, and short-term outcome of patients with type A acute dissection complicated by mesenteric malperfusion: Observations from the International Registry of Acute Aortic Dissection. Thorac Cardiovasc Surg 2013;145:385-90. https://doi.org/10.1016/j.jtcvs.2012.01.042.

8. Girdauskas E, Kuntze T, Borger MA, Falk V, Mohr FW. Surgical risk of preoperative malperfusion in acute type A aortic dissection. Thorac Cardiovasc Surg 2009;138:13639.https://doi.org/10.1016/j.jtcvs.2009.04.059.

9. Bhatti U, Kalra M, Bower Thomas C, Oderich GS, Fleming M, DeMartino RR, Heins C, Gloviczki P. Acute arterial mesenteric ischemia: contemporary single center experience over 15 years. $J$ Vasc Surg 2016; 63, Supplement: p194s-195s. https://doi.org/10.1016/j.jvs.2016.03.328. 